\title{
Analysis of the efficacy of an adjuvant-based inactivated pandemic H5N1 influenza virus vaccine
}

\author{
Ainur Nurpeisova ${ }^{1}$ - Markhabat Kassenov ${ }^{1} \cdot$ Nurkuisa Rametov $^{1} \cdot$ Kaissar Tabynov $^{1} \cdot$ Gourapura J. Renukaradhya $^{2}$. \\ Yevgeniy Volgin ${ }^{1} \cdot$ Altynay Sagymbay $^{1} \cdot$ Amanzhol Makbuz $^{3} \cdot$ Abylay Sansyzbay $^{1} \cdot$ Berik Khairullin $^{1}$
}

Received: 5 October 2018 / Accepted: 7 January 2019 / Published online: 10 February 2019

(c) Springer-Verlag GmbH Austria, part of Springer Nature 2019

\begin{abstract}
This paper describes a preclinical study analyzing the immunogenicity and protective efficacy of Kazfluvac ${ }^{\circledR}$, an adjuvantbased inactivated pandemic influenza A/H5N1 virus vaccine. In this study, laboratory animals (ferrets and mice) were vaccinated by the intramuscular or intraperitoneal route at an interval of 14 days with two doses of the vaccine containing different concentrations of influenza virus hemagglutinin (HA) protein. HA protein without adjuvant (aluminum hydroxide and Merthiolate) was used as a control. As a negative control, we utilized PBS. We assessed the protective efficacy of the candidate vaccine by analyzing the response to challenge with the influenza virus strain A/chicken/Astana/6/05 (H5N1). Our experimental results revealed substantially reduced clinical disease and an increased antibody response, as determined by hemagglutination-inhibition (HAI) test and microneutralization assay (MNA). This study showed that the candidate vaccine is safe and elicits an antigen-dose-dependent serum antibody response. In summary, we determined the optimum antigen dose in a Kazfluvac ${ }^{\circledR}$ adjuvant formulation required for induction of heightened immunogenicity and protective efficacy to mitigate $\mathrm{H} 5 \mathrm{~N} 1$ disease in experimental animals, suggesting its readiness for clinical studies in humans.
\end{abstract}

\section{Introduction}

Outbreaks of highly pathogenic avian influenza A (H5N1) virus among wild and domestic birds in Southeast Asia in 1997-2004 and its spread to other countries in Asia, Europe and Africa, coupled with putative mutations of the virus, have complicated the epidemiology of influenza considerably [1]. The emergence of a highly pathogenic influenza virus of the $\mathrm{H} 5$ subtype that is capable of infecting humans without prior adaptation and its unprecedented spread throughout the world have brought about a permanent threat

Handling Editor: Dundon.

Ainur Nurpeisova

nurai1005@gmail.com

1 Research Institute for Biological Safety Problems (RIBSP), Gvardeyskiy, Korday district, Zhambyl Region, Republic of Kazakhstan

2 Department of Veterinary Preventive Medicine, Food Animal Health Research Program, Ohio Agricultural Research and Development Center, The Ohio State University (OSU), Wooster, OH 44691, USA

3 Kazakh National Agrarian University, Almaty, Republic of Kazakhstan of a pandemic. In addition to infection of birds and destruction of flocks, this pathogen continues to circulate in Asia and cause disease in humans [2,3]. According to WHO, by 19 July 2016, avian influenza A (H5N1) virus had infected 854 people in Indonesia, Vietnam, China, Cambodia, Thailand, Azerbaijan and other countries, resulting in 450 deaths [4].

There has been uncontrolled spread of the virus in Asia, threatening the biological safety of many countries, including Kazakhstan. The first outbreak of highly pathogenic avian influenza A (H5N1) virus in Kazakhstan was reported in 2005 [5]. A strain called A/chicken/ Astana/6/05 (H5N1) was isolated from birds during an outbreak. It was determined that this strain belongs to clade 2.2 and bears substantial similarity to strains recommended by WHO for human vaccine production [6]. Influenza vaccines remain the principal means of disease prophylaxis. We at the Research Institute for Biological Safety Problems, through a reverse genetics approach, developed Kazfluvac ${ }^{\circledR}$, a pandemic avian influenza (H5N1) vaccine intended for human use. The vaccine virus strain has a number of differences in its biological properties compared to other related strains [6], and the procedure used to grow, purify, and concentrate the virus are distinct. 
This monovalent pandemic virus vaccine was produced using a technology in which inactivated whole virions are adsorbed onto an aluminum hydroxide adjuvant, $2 \%$ Alhydrogel ${ }^{\circledR}[7,8]$. The presence of aluminum hydroxide enhances the effectiveness of the vaccine and reduces the antigen dose required, which helps to minimize its reactogenicity (i.e., the ability to cause adverse reactions) and increase its safety $[6,7]$. Antigens and the adjuvant form a combination that stimulates the induction of humoral and cellular immunity, leading to the generation of protective levels of specific antibodies against influenza virus [8]. To scale up this technology, we used a new strategy to purify the virus from infected chick embryo allantoic fluid, which enabled us to obtain high levels of concentrated virus while minimizing the ovalbumin content.

The effectiveness of vaccination in preventing a pandemic viral disease is directly dependent on the quality of the vaccines utilized [9-13]. A study of the immunogenicity and protective properties of Kazfluvac ${ }^{\circledR}$ was needed to assess the vaccine's effectiveness against $\mathrm{A} / \mathrm{H} 5 \mathrm{~N} 1$ pandemic influenza virus infection in laboratory animals (ferrets, in particular). Influenza-virus-infected ferrets and humans show similarities in their symptoms, course of disease, spread of the virus in body, and humoral response $[14,15]$. However, mice are often used in studies of the immunogenicity of influenza vaccines along with ferrets [16-18]. The goal of this study was to assess the immunogenicity and protective efficacy of Kazfluvac ${ }^{\circledR}$, an inactivated adjuvant-based pandemic H5N1 influenza virus vaccine, in both ferrets and mice immunized twice with different concentrations of $\mathrm{A} / \mathrm{H} 5 \mathrm{~N} 1 \mathrm{virus}$ hemagglutinin.

\section{Materials and methods}

\section{Vaccine}

Kazfluvac ${ }^{\circledR}$, the vaccine preparation examined in this paper, is an adjuvant-based inactivated influenza strain A/AstanaRG/6:2/2009 (H5N1) vaccine in the form of a liquid suspension. This strain was produced jointly by the Research Institute for Biological Safety Problems, Ministry of Education and Science of Kazakhstan, and the Research Institute for Influenza, North-East Division of the Russian Academy of Medical Sciences, by a reverse genetics approach from the highly pathogenic A/Chicken/ Astana/6/05 (H5N1) influenza virus and the highly reproductive donor strain of A/PR/8/34 (H1N1) influenza virus (gene ratio 2:6) $[5,6]$. The study was conducted by using three experimental batches of Kazfluvac ${ }^{\circledR}$ with different concentrations of $\mathrm{A} / \mathrm{H} 5 \mathrm{~N} 1$ hemagglutinin.

\section{Animals}

To assess the immunogenicity and protective effectiveness of three experimental batches of Kazfluvac ${ }^{\circledR}$, we used 6 to 7-month-old male ferrets $(n=96)$ provided by Biotest, Czech Republic, and female BALB/c mice $(\mathrm{n}=165)$ weighing 18-22 g. Each experimental vaccine group consisted of eight ferrets and 15 mice. Experimental animals were grouped randomly ( 8 ferrets and 15 mice) to include both males and females and to achieve uniform homogeneity in terms of body weight $( \pm 20 \%)$. We collected blood samples from animals prior to the study, and they were found to be negative for serum antibodies against influenza virus.

\section{Assessment of immunogenicity and protective efficacy of the vaccine in ferrets and mice challenged with pandemic influenza virus A/H5N1}

Ferrets were vaccinated with Kazfluvac ${ }^{\circledR}$ containing different amounts of HA $(3.75 \mu \mathrm{g}, 7.5 \mu \mathrm{g}$ and $15.0 \mu \mathrm{g}$ per dose $)$ containing $2 \%$ aluminum hydroxide and Merthiolate. The animals were inoculated twice by the intramuscular route at an interval of 14 days. The control groups received 10 $\mu \mathrm{g}$ of HA or PBS containing $0.5 \mu \mathrm{g}$ of aluminum hydroxide $\left(\mathrm{Al}^{+3}\right)$. The volume of experimental vaccine and control substance was $0.5 \mathrm{ml}$ per animal. Blood samples were collected from ferrets through the subclavian vein two weeks after each immunization to determine the antibody response by hemagglutination inhibition test. To assess the protective efficacy of the vaccine, animals were infected intranasally, 14 days after the second immunization, with the strain A/chicken/Astana/6/05 (H5N1) at a dosage of $10^{6} 50 \%$ egg infectious dose per $\left(\mathrm{EID}_{50}\right) 0.5 \mathrm{ml}(0.25$ $\mathrm{ml}$ into each nostril). All of the animals were observed throughout the study period for clinical signs of disease such as sneezing, nasal discharge, changes in body temperature and weight, loss of appetite, and neurological disorders. We collected nasal wash samples and euthanized two animals from each group to sample lungs and spleen on days 3, 7 and 14 post-challenge. The viral load was determined by analyzing the infectious activity of the challenge virus in 10-day-old chick embryos to determine the $\operatorname{EID}_{50}$ value.

Groups of mice were vaccinated twice by the intraperitoneal route at an interval of 14 days with three doses of inactivated virus containing $2.5 \mu \mathrm{g}, 5 \mu \mathrm{g}$ or $10 \mu \mathrm{g}$ of HA and $2 \%$ aluminum hydroxide and Merthiolate. The control groups received PBS without adjuvant as a negative control. We sampled blood from the tail vein two weeks after the second immunization to assess the antibody response 
by HAI and MNA. To assess the protective efficacy of Kazfluvac ${ }^{\circledR}$, mice were challenged with strain A/chicken/ Astana/6/05 (H5N1), which causes lethal influenza in mice. Animals were challenged intranasally, under anesthesia, with $30 \mu \mathrm{l}$ of virus at a dose of $10^{5.5} \mathrm{TID}_{50}$ per animal. Mice were examined throughout the study period for any changes in body weight, level of activity, loss of appetite, and neurological disorders. We sampled the lungs, spleen and brain on the day of the death of the mice and determined the viral load by examining infectious influenza virus activity in 10-day-old chick embryos and determining the $\mathrm{EID}_{50}$ value as described previously [19].

\section{Viral infectivity}

We assessed the infectivity of the H5N1 challenge virus using a standard 10-fold dilution of test samples in PBS (from $10^{-1}$ to $10^{-10}$ ) inoculated into the allantoic cavity of 10-day-old chicken embryos. The embryos were incubated at $33{ }^{\circ} \mathrm{C}$ and a relative air humidity of $55 \%$ for 3 days. A hemagglutination test was performed to confirm the presence of the virus in chicken embryos, and the infectious titer was calculated at $\log 10 \mathrm{EID}_{50} / 0.2 \mathrm{ml}$ of inoculum using the Reed-Muench method [22]. All research with the pandemic virus was conducted in a biosafety level 3 environment.

\section{Assessment of the humoral response in animals}

\section{Hemagglutination inhibition (HAI) test}

The test was performed by the standard method using the avian influenza strain A/chicken/Astana/6/05 (H5N1) virus as the antigen. We removed nonspecific inhibitors by treating blood samples with a receptor-destroying enzyme from Vibrio cholerae (Denka Seiken Co. Ltd., Japan) according to the user instructions and heat treating the sera at $56{ }^{\circ} \mathrm{C}$ for 30 minutes.

\section{Microneutralization assay (MNA)}

For the assay the test sera were diluted in Eagle medium (1/10-1/1280) in round-bottom plates containing TPCK trypsin (Sigma, USA) at a concentration of $2 \mu \mathrm{l} / \mathrm{ml}$. An equal volume of virus $\left(100 \mathrm{TCID}_{50}\right.$ in $\left.50 \mu \mathrm{l}\right)$ was added to the serum, and the plates were incubated at $37^{\circ} \mathrm{C}$ for 2 hours. One hundred $\mu 1$ of the mixture was transferred to a plate (NUNC, Denmark) seeded with a MDCK cell monolayer containing $50 \mu \mathrm{l}$ of Eagle medium with TPCK trypsin $(2 \mu \mathrm{g} / \mathrm{ml})$ in each well. Two control samples were included in each plate: a cell control (CC, four wells with diluent without virus or serum) and a virus dose control ( $\mathrm{CV}$, four wells containing virus). The plates were kept in a $\mathrm{CO}_{2}$ incubator at $37^{\circ} \mathrm{C}$ for 48 hours. After incubation, the medium was removed and the cell monolayer was fixed with cold $80 \%$ acetone for 20 minutes and washed with PBS. We performed an ELISA test on the plates to detect infected cells using virus-specific monoclonal antibodies (mAbs). Plates were washed twice with PBS, and $100 \mu \mathrm{l}$ of MAb Px diluted in PBS containing 5\% skim milk $(1 / 4,000)$ was added to each well. The plates were incubated at room temperature for 1 hour and washed four times with PBS, and $100 \mu \mathrm{l}$ of substrate mixture $\left(0.02 \% \mathrm{H}_{2} \mathrm{O}_{2}\right.$ and $0.1 \mathrm{mg}$ of 3,3',5,5' -tetramethylbenzidine [Sigma, USA] per ml in $0.1 \mathrm{M}$ acetate-citrate buffer [pH 5.0] was added. The reaction was stopped after 15 minutes by adding $50 \mu \mathrm{l}$ of $2 \mathrm{~N} \mathrm{H}_{2} \mathrm{SO}_{4}$ in each well, and the optical density at $450 \mathrm{~nm}$ $\left(\mathrm{OD}_{450}\right)$ was measured using an Anthos-2010 photometer (Austria). The virus-neutralizing titer was calculated as the endpoint dilution of antibody (using OD450) at which the infectivity remained below the threshold value (TV), computed by the following formula:

$T V=\frac{O D 450 C V-O D 450 C C}{2}+O D 450 C C$,

where: $\mathrm{OD}_{450} \mathrm{CV}$ is the average $\mathrm{OD}_{450}$ value in control wells containing virus working dose, and $\mathrm{OD}_{450} \mathrm{CC}$ is the average $\mathrm{OD}_{450}$ value in control uninfected wells

\section{Statistical analysis}

Statistical analysis of all experimental data was performed using Graphpad Prism Software version 6.0 (GraphPad Software Inc., CA, USA). We assessed immunogenicity in the test groups by determining the geometric mean titer (GMT) of antibodies with a confidence interval of 95\%. Means are reported with standard errors (SEM). Significance between groups was determined by two-way ANOVA, and significance was set at $P<0.05$. We used a one-way analysis of variance (Dunnett's test) to compare dose-dependent changes in the body weight of the experimental animals in the test groups. We analyzed the survival of challenged animals using the Logrank test. All other data operations and statistical computations were conducted using StatSoft software, version 12.

\section{Statement of ethics}

Animal procedures were performed in accordance with national and international laws and guidelines on laboratory animal handling. The protocol was approved by the Committee on the Ethics of Animal Experiments of the Research Institute for Biological Safety Problems (Permit Number 0909/25). 


\section{Results}

\section{Assessing the immunogenicity and protective efficacy of the vaccine in ferrets}

No deviations in the somatic or neurological status of vaccinated animals in the test or control groups were observed during the observation period after two immunizations. The body weight of animals in the test and control groups did not differ significantly over time. The gain in body weight on day 14 after the first and second immunization in animals vaccinated with Kazfluvac ${ }^{\circledR}$ and control agents varied from $0.1 \%$ to $3.5 \%$ (Fig. 1). The body weight change in animals of the test group on the sixth to seventh day after the first and second immunizations was comparable to that in the control group. In both groups, the body weight increased by 14 days, suggesting that the vaccine did not cause any loss in body weight in ferrets. The body temperature of the test and control groups of ferrets was normal for 14 days after the first and second immunizations with no major fluctuations (Fig. 1).

(a)

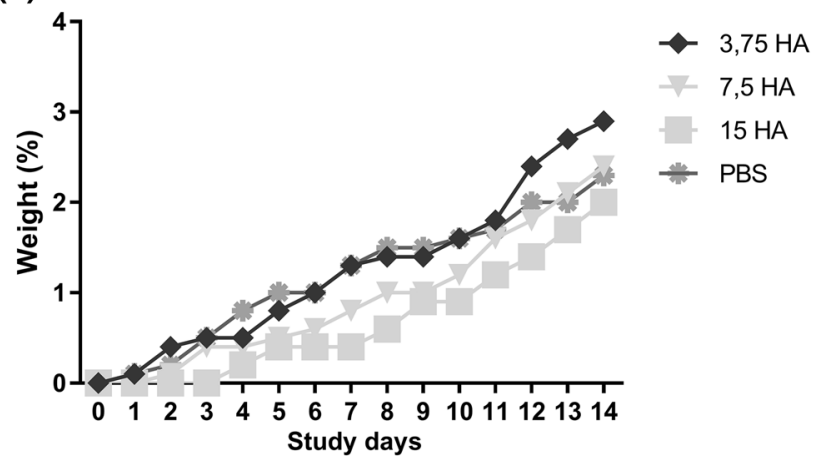

(c)

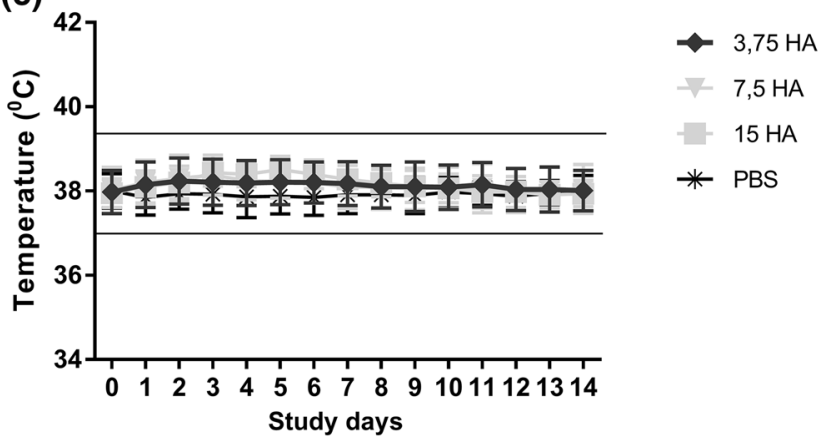

Fig. 1 Analysis of changes in the body weight and temperature of vaccinated ferrets. (A and B) Changes in the body weight of ferrets immunized with Kazfluvac ${ }^{\circledR}$ vaccine after the first (A) and second (B) immunization, shown as the percentage of the initial weight $(0 \%)$ before the experiment. Analysis of daily readings of body tempera-
A comparison of antibody GMT values after the first immunization with different HA concentrations showed variations between the test groups compared to controls, in particular, significant differences were observed between ferrets immunized with $3.75 \mu \mathrm{g}(P=0.002), 7.5 \mu \mathrm{g}(P=$ $0.0001)$, and $15.0 \mu \mathrm{g}(P=0.0001)$ of HA compared to the PBS control, suggesting that a single immunization of ferrets using different amounts of antigen promoted a dosedependent antibody response. However, the difference in the GMT values of animals immunized with $7.5 \mu \mathrm{g}$ and $15.0 \mu \mathrm{g}$ HA per dose were not significantly different (Fig. 2A). The second immunization induced a significant $(P<0.05)$ GMT value difference in all of the experimental groups when compared to the first immunization and the control groups of ferrets (Fig. 1B). These results demonstrated that, irrespective of antigen dose and adjuvant $\left(\mathrm{Al}^{+3}\right)$, a booster dose of Kazfluvac ${ }^{\circledR}$ provided a stronger immune response (Fig. 2). Ferrets in the negative-control group either did not develop antibodies or their antibody levels remain lower than 1:10.

During the 14-day observation period, clinical signs were observed in $75 \%(6 / 8)$ of the animals in the negative control group starting on day 3 . These signs include sneezing, reduced activity, loss of appetite, difficulty in breathing, and

(b)

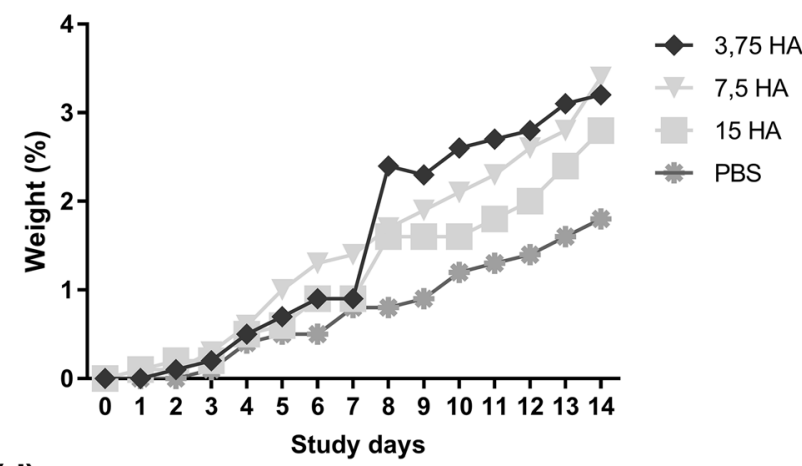

(d)

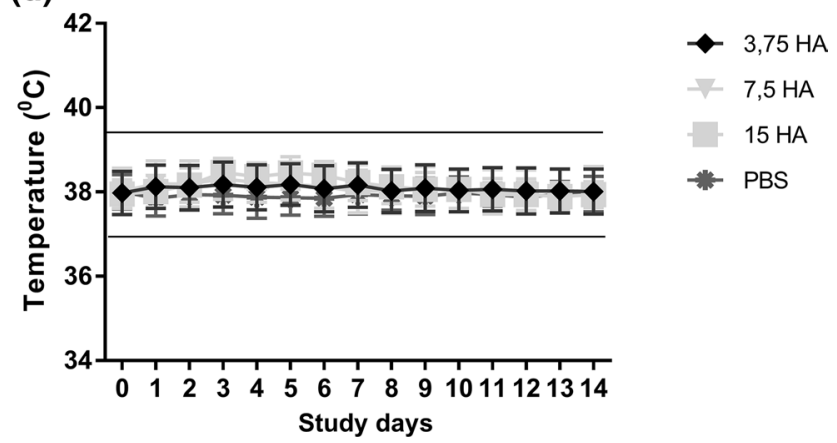

tures in ferrets (with standard deviation) of the test and control groups for 14 days after the first (C) and second (D) vaccination did not identify any deviations from the normal values. The observation period totaled 14 days and the standard deviation (SD) of the mean body temperature for each group is indicated by error bars 
Fig. 2 HAI analysis of

Kazfluvac $^{\circledR}$-vaccinated ferrets.

The HA concentration in the vaccine dose $(3.75,7.5$ and 15.0 $\mu \mathrm{g}$ ) was compared with that of a control group that received PBS with aluminum hydroxide. Each experimental group had eight ferrets. Analysis of GMT differences was performed using Dunnett's multiple comparisons test with a confidence interval of $95 \%$ after each vaccination. HAI titers were analyzed after (A) the first immunization and (B) the second immunization (a)

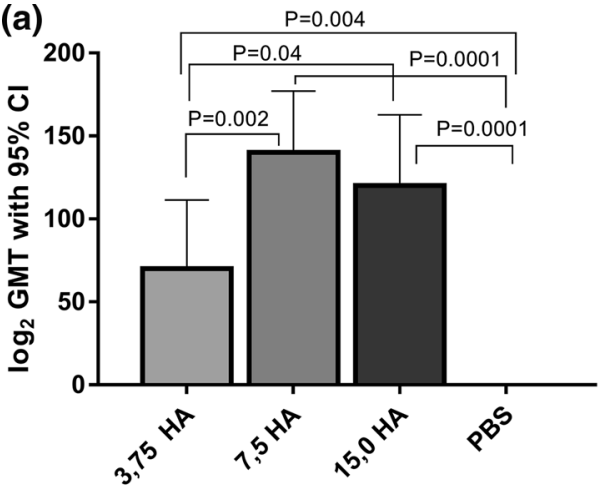

Ferret groups

Vaccine (HA 3.75)

Vaccine (HA 7.5)

Vaccine (HA 15.0) PBS

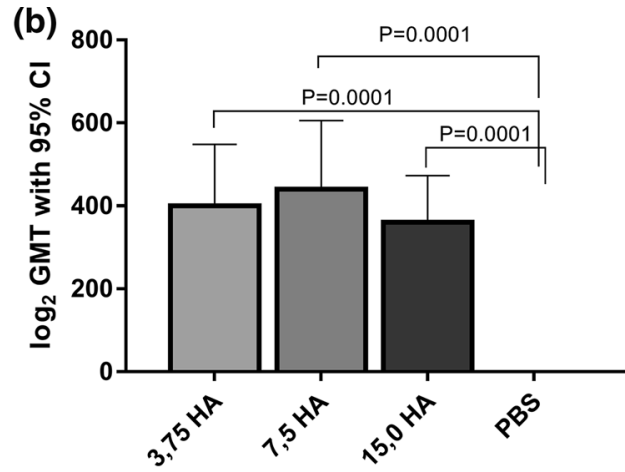

\begin{tabular}{|c|c|c|c|}
\hline \multicolumn{2}{|c|}{ First immunization } & \multicolumn{2}{|c|}{ Second immunization } \\
\hline GMT & $95 \% \mathrm{CI}$ & GMT & $95 \% \mathrm{CI}$ \\
\hline 61.6 & $40.08-94.94$ & 400 & $291-497.6$ \\
\hline 134.5 & $102.9-175.9$ & 440 & $307.5-560.1$ \\
\hline 113.1 & $83-154.2$ & 360 & $284.3-428.3$ \\
\hline & & & \\
\hline
\end{tabular}

a watery discharge from the nose and eyes that over time became dense and formed a crust around the nasal turbinate. One other clinical sign that was noted was a steady loss of body weight.

A statistical analysis using StatSoft showed that there were significant differences in body weight at each vaccination (first and second) when compared to the time of challenge $(P<0.05)$. We compared the body weight of animals after the first vaccination and after the challenge and found significant differences from days 1 to 4 after challenge, with the significance level at $P=0.001-0.02$. We compared the body weight of animals after the second vaccination and post-challenge and found a significant difference from day 1 to 3 post-challenge, with the significance level at $P$ $=0.02-0.04$. (Fig. 2 and 3). In spite of an increased trend towards loss of weight on day 3 after challenge, ferrets that had received two immunizations gained in body weight on day 6 , with the weight gain depending on the amount of HA (8.0\% gain with $3.75 \mu \mathrm{g}$ of HA, $9.5 \%$ with $7.5 \mu \mathrm{g}$, and $6.8 \%$ with15.0 $\mu \mathrm{g})$.

A significant difference $(P<0.05)$ in the body temperature of the test and control groups was observed on day 6 . The average body temperature in test-group animals exceeded the physiological limit $\left(39.0{ }^{\circ} \mathrm{C}\right)$ only on days $4-5$, whereas in the control group it was high from day 1 after challenge and remained unchanged until day 8 . The body temperature of vaccinated ferrets remained within the normal range (Fig. 3) throughout the entire observation period. The benchmark value used for the average body temperature was the average body temperature readings recorded for 3 days pre-challenge. Statistical analysis of variance of the body temperature between the test and control groups showed a significant difference $(P=0.004)$. On day 2 postchallenge, there was a statistical difference based on the number of vaccinations $(P=0.006)$ and the different HA concentrations in the vaccine used compared to the control group, which remained higher until day $13(P<0.05)$.

The presence of virus was detected in nasal wash samples in $100 \%$ of animals in the control group at 3 and 7 days after challenge, while in immunized animals, virus was detectable 3-7 days after challenge, and the viral load was 1000 times lower than in the control group. We also examined the viral load in the internal organs of ferrets to assess the extent of viral invasion in their bodies. On days 7 and 14 after challenge, two animals from each group were euthanized, and the virus load in their lungs and spleen was measured. In the immunized group, reduced virus titers were observed in the lung tissues at day 7 , and by day 14 , the virus was undetectable. Significant differences in virus load were observed in the lungs at days 7 and $14(P=0.029)$, and the difference between virus accumulation in the spleen and nasal wash samples was also significant $(P<0.05)$. Two-way analysis of variance to evaluate the shared impact of the time (number of days) and the different HA concentrations in the vaccine showed a significant difference $(P=0.02)$.

\section{Assessing the immunogenicity and protective efficacy of the vaccine in mice}

The general condition of the mice after each immunization with Kazfluvac ${ }^{\circledR}$ and control agents was normal. The GMT values for HAI and MNA antibodies in sera of mice that received two immunizations was relatively high and was dependent on the amount of HA in the vaccine formulation. The GMT values in twice-immunized mice measured by HAI and MNA are presented in Table 1.

An analysis of HAI results using the Tukey multiple comparison test indicated statistically significant differences $(P$ $=0.0004)$ in the GMT of mice immunized with Kazfluvac ${ }^{\circledR}$ containing $2.5 \mu \mathrm{g}$ and $10.0 \mu \mathrm{g}$ of HA. The group of mice 

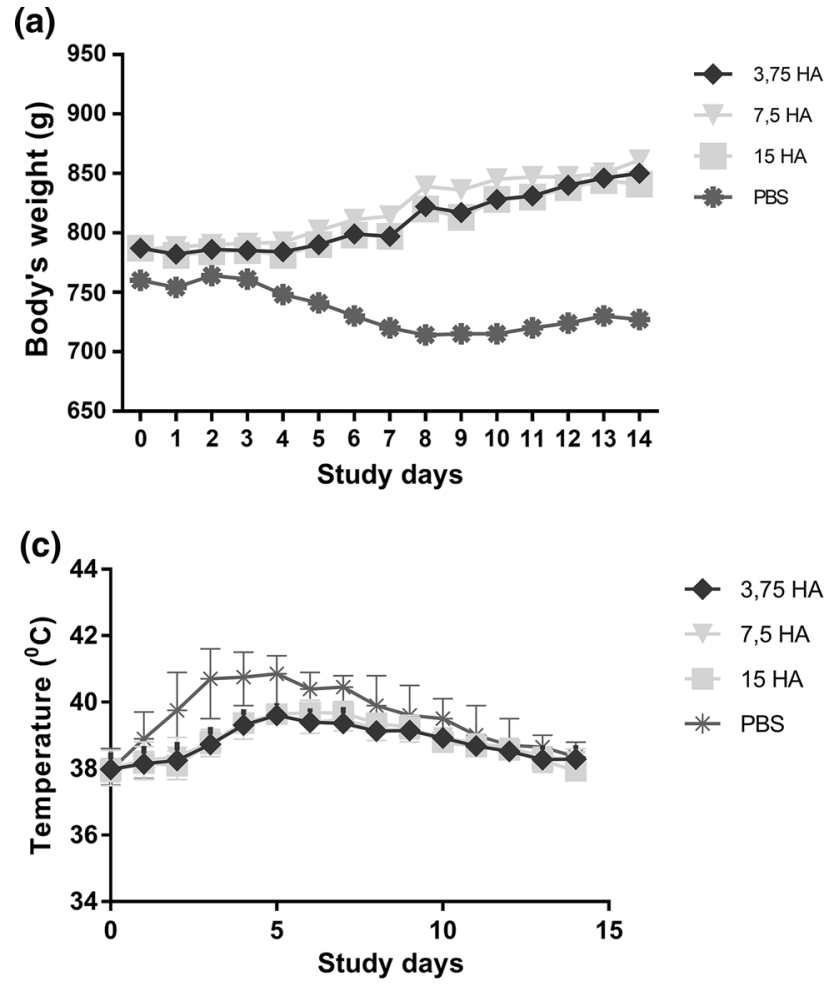

Fig. 3 Analysis of clinical observation of vaccinated ferrets postchallenge. Body weight, body temperature, and titers of infectious influenza virus in certain organs of test- and control-group animals 14 days after challenge infection with A/chicken/Astana/6/05 (H5N1) were determined. Each experimental group had eight ferrets. (A) Body weight in grams (average). (B) Analysis of the percentage deviation in the body weight of ferrets. Weight gain depended on the HA

Table 1 Hemagglutination-inhibition test and microneutralization assay results

\begin{tabular}{llllll}
\hline Mouse group & \multicolumn{2}{l}{ HAI } & & \multicolumn{2}{l}{ MNA } \\
\cline { 2 - 3 } \cline { 5 - 6 } \cline { 5 - 6 } & GMT & $95 \%$ CI & & GMT & $95 \%$ CI \\
\hline Vaccine (HA 2.5) & 60.6 & $22.73-161.8$ & & 121.3 & $56.16-261.8$ \\
Vaccine (HA 5.0) & 91.9 & $20.0-160.0$ & & 278.6 & $67.73-1146$ \\
Vaccine (HA 10.0) & 278.6 & $135.6-572.4$ & & 422.2 & $263.5-676.5$ \\
SP (HA 10.0) & 45.95 & $22.36-94.4$ & & 211.1 & $131.8-338.3$ \\
PBS & - & - & & - \\
\hline
\end{tabular}

HAI, hemagglutination-inhibition test; MNA, microneutralization assay; GMT, geometric mean titer; HA, hemagglutinin; SP, semi-finished product of the vaccine Kazfluvac ${ }^{\circledR}$

immunized with a vaccine containing $10.0 \mu \mathrm{g}$ of HA also showed a significant difference $(P=0.01)$ compared with the group immunized with $5.0 \mu \mathrm{g}$ of HA. The GMT of animals immunized with vaccine containing $10.0 \mu \mathrm{g}$ of HA differed significantly $(P=0.001)$ from those of animals

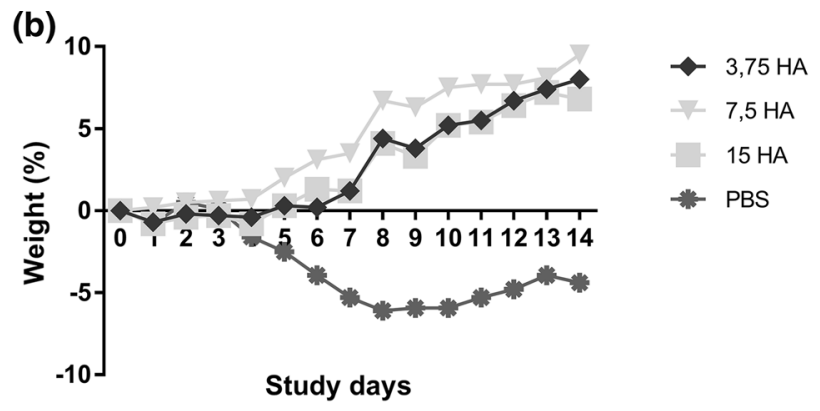

(d)

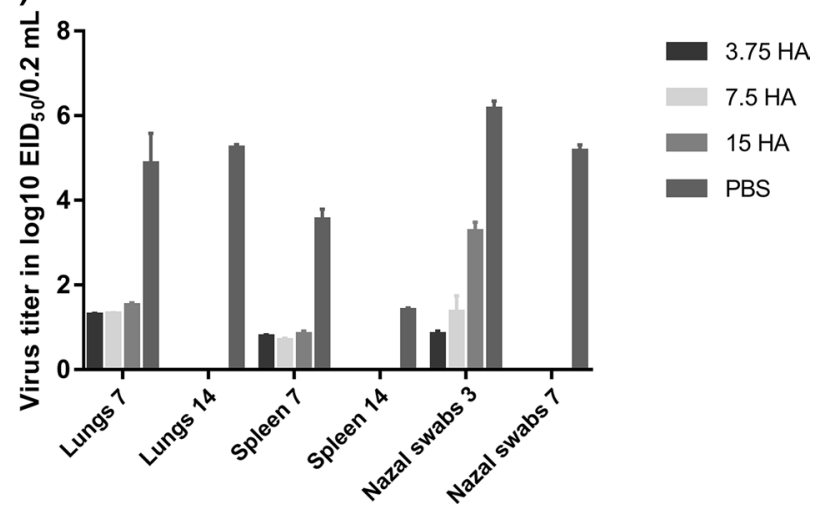

concentration in the vaccine: $8.0 \%(3.75 \mu \mathrm{g}), 9.5 \%(7.5 \mu \mathrm{g})$ and $6.8 \%$ $(15.0 \mu \mathrm{g})$. (C) Fluctuations in the body temperature of ferrets. The observation period was 14 days, and the standard deviation (SD) for the mean body temperature of each group is indicated by error bars. (D) Infectious influenza virus titer in certain organs (lungs, spleen and nasal cavity) of test- and control-group ferrets

immunized with $10.0 \mu \mathrm{g}$ of control HA without adjuvant and those that were given PBS $(P=0.001)$ (Fig. 4a).

We confirmed, using MNA, that a double vaccination with Kazfluvac ${ }^{\circledR}$ in three different dosages provided a $100 \%$ seroconversion in all animal groups, including the one immunized with control HA (Fig. 4b). The GMT in mice immunized with a vaccine containing $2.5 \mu \mathrm{g}$ of HA was significantly different $(P=0.03)$ from that of mice vaccinated with $10.0 \mu \mathrm{g}$ of HA. The group of mice vaccinated with 5.0 $\mu \mathrm{g}$ of HA per dose had a significantly different GMT $(P=$ $0.004)$ from the group that received PBS. Also, a significant difference $(P=0.001)$ was observed between groups of mice vaccinated with $10.0 \mu \mathrm{g}$ of HA and the control group.

The protective efficacy of the vaccine after two immunizations was tested by challenging mice with influenza virus strain A/chicken/Astana/6/2005 (H5N1) and monitoring their general health, neurological state, body weight and survival in comparison to the control group for 14 days (Fig. 5). The average body weight in the groups vaccinated with $2.5 \mu \mathrm{g}$ and $5.0 \mu \mathrm{g}$ of HA per dose decreased 
Fig. 4 Immune response in mice vaccinated with Kazfluvac ${ }^{\circledR}$.

Vaccine preparations containing $2.5 \mu \mathrm{g}, 5.0 \mu \mathrm{g}$ or $10.0 \mu \mathrm{g}$ of HA with adjuvant or control agents were injected twice at an interval of 14 days, and antibody titers were determined by HAI (A) and MNA (B). GMT values of antibodies were dependent on the amount of HA in the vaccine formulation. The analysis of GMT values was performed using the Turkey multiple comparison test

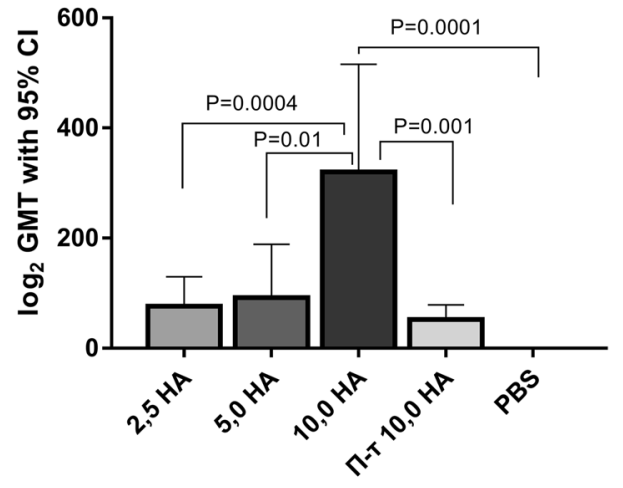

(a) HAl antibodies

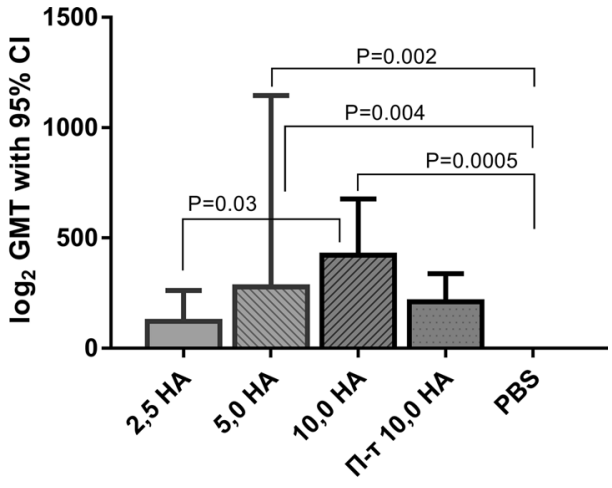

(b) MN antibodies

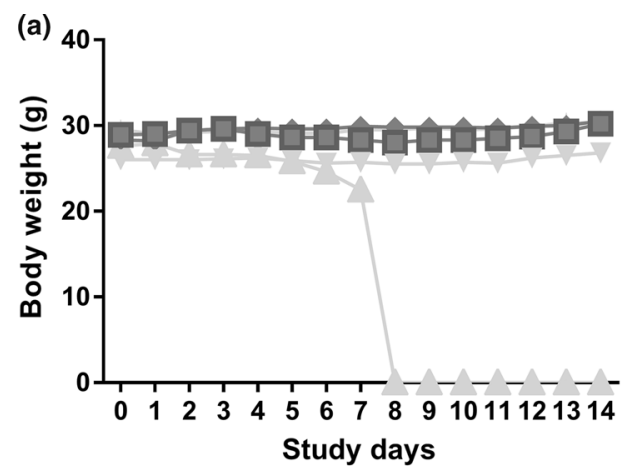

(c)

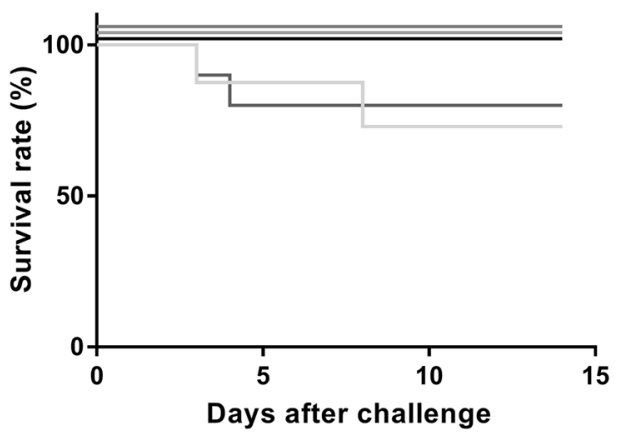

Fig. 5 Dynamics of body weight gain in vaccinated and challenged mice. Animals were challenged 14 days after the second vaccination with $\mathrm{A} /$ chicken/Astana/6/05 (H5N1). (A) Average weight in grams. (B) Percentage variance in body weight. (C) Survival rate of challenged mice over a period of 14 days. Survival curves of all immu-

considerably from days 5 to 12 . The group inoculated with control HA showed a declining trend from days 1 to 10 . In spite of significant loss of body weight at the beginning, by the end of the observation period, the animals in all test groups, unlike the control group, gained weight. Starting on day 8 , fatalities were observed in the control group (Fig. 5A and B). None of the mice vaccinated with Kazfluvac ${ }^{\circledR}$, irrespective of the antigen load, lost weight during the whole

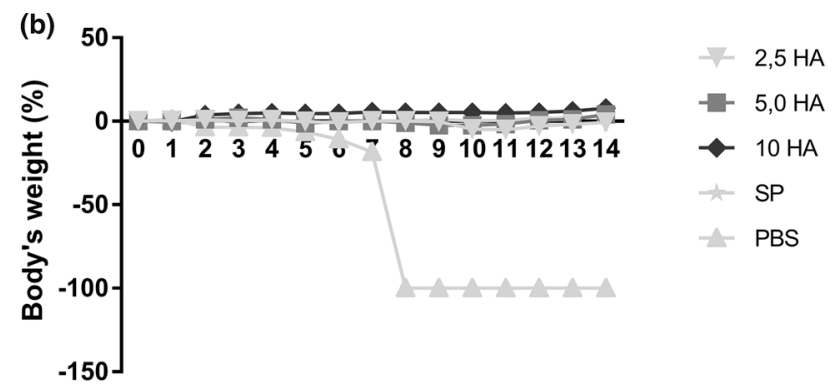

Study days

(d)

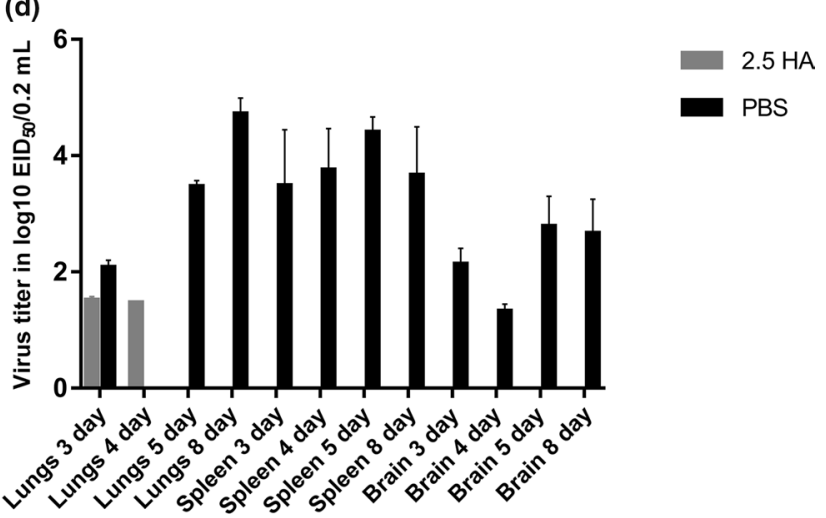

nized groups compared to control group. (D) Demonstration of a protective effect of Kazfluvac ${ }^{\circledR}$ in vaccinated animals in terms of the viral load in the lungs, spleen, and brain on the day of death due to challenge virus infection. We determined the virus load in dead mice of the control group and in those immunized with $2.5 \mu \mathrm{g}$ of HA

observation period of 14 days, and in fact gained 0.6-3.5 g by the end of the experiment. We observed a larger gain in body weight in mice immunized with larger doses of the vaccine. A similar pattern was observed in immunized mice in the uninfected control group, but their body weight at the end of the experiment had increased by a negligible amount $(0.8 \mathrm{~g})$. The maximum average body weight was observed in the group vaccinated with $10.0 \mu \mathrm{g}$ of HA per dose was 29.7 
$\mathrm{g}$, whereas the maximum average weight in the control group was $22.5 \mathrm{~g}$. Weight loss in the control group started on day 3 and reached a peak on day $7(5.1 \mathrm{~g})$ due to marked clinical signs including loss of appetite. An analysis of the body weight pattern in challenged mice (one-way analysis, Dunnett's test) showed significant differences in all test groups versus the control group $(P=0.0001)$.

We demonstrated a protective effect of Kazfluvac ${ }^{\circledR}$ on animals challenged with influenza virus, except in the group immunized with $2.5 \mu \mathrm{g}$ of $\mathrm{HA}$, in which one animal died on the third day and one on the fourth day of observation (Fig. 5C). The unimmunized control group had a mortality rate of $50 \%$, with deaths of mice occurring from day 3 to day 8 after challenge. Before death, animals in this group exhibited signs of hypodynamia, disheveled hair, tachypnea, and lack of appetite. Necropsy of these mice revealed signs of acute congestive hyperemia of the lungs, subcutaneous tissues, liver, and kidneys with acute endoenteritis and petechial hemorrhages in the mucosa of the small bowel in some mice, all of which are typical of acute infection. We analyzed the survival curves of all test groups versus the control group using the logrank test and found statistically significant differences $(P=0.009)$ (Fig. 5C).

We determined the virus load in dead mice from the control group and the group immunized with $2.5 \mu \mathrm{g}$ of HA by sampling their lungs, spleen, and brain using 10-day-old chicken embryos for titration. The amount of infectious virus in mice immunized with $2.5 \mu \mathrm{g}$ of HA was insignificant, from $1.45 \pm 0.22 \log \mathrm{EID}_{50} / \mathrm{ml}$ to $1.50 \pm 0.33 \log \mathrm{EID}_{50} / \mathrm{ml}$, and it was found only in the lung samples (Fig. 5D). In control animals, we observed a generalized course of infection with high titers of virus detected in all body organs, with the largest amount of virus accumulating in the lungs (from $1.95 \pm 0.28 \log \mathrm{EID}_{50} / \mathrm{ml}$ to $5.00 \pm 0.42 \log \mathrm{EID}_{50} / \mathrm{ml}$ ). All control mice were dead on the tenth day after challenge due to generalized infection of vital organs (Fig. 5D).

\section{Discussion}

The high prevalence of $\mathrm{H} 5 \mathrm{~N} 1$ influenza virus in domestic and wild birds in Asia and its extremely high mortality in infected humans warrant the development of a potent vaccine and the production of national vaccine stocks to prevent potential pandemics $[20,21]$. According to WHO recommendations, each country should develop its own vaccines and enlarge its production capacities. In recent years, the Research Institute for Biological Safety Problems (RIBSP) of the Ministry of Education and Science of Kazakhstan has conducted studies directed towards the development of vaccines for pandemic and seasonal influenza to be used in Kazakhstan's disease prevention programs. The quality of RIBSP's Kazfluvac ${ }^{\circledR}$ is not inferior to that of European or
American vaccines. Reports from 2004-2008 have described newly developed inactivated whole-virion vaccines for avian influenza A (H5N1) virus derived from the recombinant strain NIBRG-14 (Biken, Japan), a recombinant H5N1strain (GSK Biologicals, Canada-Germany), and recombinant strain NIBRG-14 (Nobilon International BV, the Netherlands), as well as a surface-inactivated antigen vaccine from strain A/Vietnam/1203/2004/ (H5N1) (Novartis Vaccines \& Diagnostics [V\&D]) and others [11, 22-25]. Conducting all stages of research to develop the vaccine in line with international standards, RIBSP from Kazakhstan produced its vaccine at almost the same time as the EU, USA and Russia produced theirs [26].

Mouse and ferret models are widely used for studies of the pathogenicity of avian influenza viruses in mammals, and their use can usually help to predict the pandemic potential of such viruses and aid in the design of advanced methods for prevention and potential treatment of pandemic influenza virus H5N1 infections [16, 27-35]. We examined the protective effect and safety of Kazfluvac ${ }^{\circledR}$ containing different amounts of antigen in ferrets and mice.

The protection provided by influenza vaccines is based on the induction of virus-neutralizing antibodies, mainly against the surface hemagglutinin protein [20,31]. Previous studies have indicated that adjuvant-based vaccines possess a higher level of immunogenicity than non-adjuvant-based ones [34-38]. The adjuvant used in Kazfluvac ${ }^{\circledR}$ is aluminum hydroxide. The use of this adjuvant increases the immunogenicity of vaccines to a great extent and allows the antigen dose to be reduced, which in turn reduces the vaccine's reactogenicity. The results of our double intramuscular immunization study showed that the vaccine induced serum antibodies in $100 \%$ of animals.

In the course of the study, we optimized the dosage of Kazfluvac ${ }^{\circledR}$. Using an HAI test, we determined that the vaccine preparations with lower concentrations of HA per dose (3.75 $\mu \mathrm{g}$ and $7.5 \mu \mathrm{g}$ ) were sufficient to protect ferrets against a challenge infection. A similar antibody response in mice was induced by Kazfluvac ${ }^{\circledR}$ with an antigen load of $10.0 \mu \mathrm{g}$ per dose, as determined by HAI and MNA tests.

Homologous challenge has been used in virus efficacy trials of influenza candidate vaccines to study protectiveness of the vaccine in outbred animals. The spread of H5N1 disease may become uncontrollable throughout a vast area and may threaten the biosafety of a large number of countries, including the Republic of Kazakhstan. In selecting a vaccine strain to develop the Kazfluvac ${ }^{\circledR}$ vaccine against H5N1, we conducted a phylogenetic analysis of influenza virus strains A/domestic goose/Pavlodar/1/05, A/chicken/Astana/6/05, A/chicken/ NKR/5/05 (H5N1), which were isolated from infected birds in 2005 during epizootic outbreaks in Kazakhstan [5, 39]. All of these strains are endemic in the Republic of Kazakhstan. A comparison of the amino acid sequences of the HA genes of 
these three strains and those of vaccine strains of H5N1 recommended by WHO for human vaccine production showed that the Kazakhstani strain most similar to recommended strains is $\mathrm{A} /$ chicken/Astana/6/05 (H5N1) [5, 39]

Influenza virus strain A/chicken/Astana/6/05 (H5N1) was therefore selected as the source of surface antigens to produce a vaccine candidate strain that is appropriate for use in Kazakhstan, using reverse genetics. The HA of this virus contains a motif of repetitive basic amino acids in the cleavage site of HA, which determines its high virulence in humans. Ferrets and a group of mice receiving a vaccine preparation containing 5.0 and $10.0 \mu \mathrm{g}$ of HA per dose were protected against challenge with the homologous influenza virus, A/chicken/ Astana/6/05 (H5N1), as indicated by the absence of clinical signs of disease and weight loss [5, 35]. Human-equivalent doses did not cause any clinical signs or loss of body weight in ferrets. Our study showed that Kazfluvac® provides a high degree of protection and prevents clinical signs and mortality in vaccinated ferrets. The correlation in ferrets between body weight and the amounts of HA in the preparation is another indication of the protective efficacy of the vaccine. The loss of body weight in vaccinated ferrets did not exceed $0.8 \%$, whereas unimmunized control ferrets lost $5.9 \%$ of their body weight on days 3-6 after challenge. It should be noted that a similar degree of protection was demonstrated in ferrets using a pandemic H1N1 vaccine developed earlier by RIBSP [40].

Experiments in mice showed that all of the dosages of Kazfluvac $^{\circledR}$ that were tested, when co-administered with or without adjuvant provided high levels of protection in mice that were challenged with epizootic virus and prevented clinical signs and mortality. Our results demonstrate that the adjuvant-based Kazfluvac ${ }^{\circledR}$ is an effective vaccine for H5N1 influenza that induces a specific immune response to H5N1 in animal models. The results of this study and previous research findings allow us to recommend Kazfluvac ${ }^{\circledR}$ for clinical studies.

Acknowledgements We thank Zh. Kydyrbayev, Y. Kozhamkulov, D. Inkarbekov, Kairat Tabynov, and N. Assanzhanova, employees of the Research Institute for Biological Safety Problems, for their assistance in this study. The study was funded by the Science Committee of the Ministry of Education and Science of the Republic of Kazakhstan. The authors express deep gratitude to the Research Institute of Influenza (St. Petersburg, Russia) for providing the vaccine strain A/ AstanaRG/6:2/2009 (H5N1) which was constructed by reverse genetics from the highly pathogenic avian influenza virus strain $\mathrm{A} /$ chicken/ Astana/6/05 of $(\mathrm{H} 5 \mathrm{~N} 1)$ and the highly reproductive influenza virus donor strain A/PR/8/34 (H1N1).

\section{References}

1. Capua I, Alexander DJ (2009) Avian influenza infection in birds: a challenge and opportunity for the poultry veterinarian. Poult Sci 88(4):842-846. https://doi.org/10.3382/ps.2008-00289
2. Beigel JH, Farrar J, Han AM et al (2005) Avian influenza A (H5N1) infection in humans. N Engl J Med 353(13):1374-1385. https://doi.org/10.1056/NEJMra052211

3. Fleming DM, Elliot AJ (2005) The impact of influenza on the health and health care utilisation of elderly people. Vaccine 23:19. https://doi.org/10.1016/j.vaccine.2005.04.018

4. World Health Organization (2016) Cumulative number of confirmed human cases of avian influenza $\mathrm{A} /(\mathrm{H} 5 \mathrm{~N} 1)$ reported to WHO. http://www.who.int/influenza/human_animal_interface/ H5N1_cumulative_table_archives/en/. Accessed 20 June 2018

5. Kydyrbaev ZhK, Tabynov KK, Khairullin BM (2015) Vysokopatogennyj gripp ptic: rasprostranenie $\mathrm{v}$ Kazahstane i razrabotka sredstv specificheskoj profilaktiki [Highly pathogenic avian influenza: distribution in Kazakhstan and development of means of specific prevention]. Almaty (in Russian)

6. Sansyzbay AR, Erofeeva MK, Khairullin BM et al (2013) Inactivated and adjuvanted whole virion clade $2.2 \mathrm{H} 5 \mathrm{~N} 1$ (A/chicken/ Astana/6/05) influenza vaccine is safe and immunogenic in a single dose in humans. Clin Vaccine Immunol 20(8):1314-1319. https://doi.org/10.1128/CVI.00096-13

7. Stukova M, Mamadaliyev S, Sandybayev N et al (2011) Basic results of development of a production technology and control of a pandemic influenza A/H5N1 vaccine. Influenza Other Respir Viruses 5(1):350-353

8. Nurpeysova A, Khairullin B, Kassenov M et al (2011) Preclinical testing of Kazfluvac ${ }^{\circledR}$, a vaccine against pandemic influenza A/ H5N1v. J Pharm Biomed Sci 1(5):108-112

9. Langley JM, Frenette L, Ferguson L et al (2010) Safety and crossreactive immunogenicity of candidate AS03-adjuvanted prepandemic H5N1 influenza vaccines: a randomized controlled phase 1/2 trial in adults. J Infect Dis 201(11):1644-1653. https://doi. org/10.1086/652701

10. Li H, Nookala S, Re F (2007) Aluminum hydroxide adjuvants activate caspase- 1 and induce IL- $1 \beta$ and IL-18 release. J Immunol 178(8):5271-5276. https://doi.org/10.4049/jimmunol.178.8.5271

11. Cox JC, Coulter AR (1997) Adjuvants-a classification and review of their modes of action. Vaccine 15(3):248-256. https:// doi.org/10.1016/S0264-410X(96)00183-1

12. Lambert LC, Fauci AS (2010) Influenza vaccines for the future. $N$ Engl J Med 363(21):2036-2044. https://doi.org/10.1056/NEJMr a1002842

13. Bodewes R, Osterhaus AD, Rimmelzwaan GF (2010) Targets for the induction of protective immunity against influenza a viruses. Viruses 2(1):166-188. https://doi.org/10.3390/v2010166

14. Treanor JJ, Campbell JD, Zangwill KM et al (2006) Safety and immunogenicity of an inactivated subvirion influenza $\mathrm{A}$ (H5N1) vaccine. N Engl J Med 354(13):1343-1351. https://doi. org/10.1056/NEJMoa055778

15. Nichol KL, Treanor JJ (2006) Vaccines for seasonal and pandemic influenza. J Infect Dis 194(2):111-118. https://doi. org/10.1086/507544

16. Monto AS (2006) Vaccines and antiviral drugs in pandemic preparedness. Emerg Infect Dis 12(1):55. https://doi.org/10.3201/ eid1201.051068

17. Langlois I (2005) Viral diseases of ferrets. Vet Clin N Am Exot Anim Pract 8(1):139-160. https://doi.org/10.1016/j. cvex.2004.09.008

18. Belser JA, Katz JM, Tumpey TM (2011) The ferret as a model organism to study influenza A virus infection. Dis Model Mech 4(5):575-579. https://doi.org/10.1242/dmm.007823

19. Palmer D, Dowdle W, Coleman M et al (1975) Advanced laboratory techniques for influenza diagnosis. Immunology series no. 6 . Part 2, procedural guide. US Department of Health Education and Public Health Service, Atlanta, pp 25-62

20. Huprikar J, Rabinowitz S (1980) A simplified plaque assay for influenza viruses in Madin-Darby kidney (MDCK) cells. 
J Virol Methods 1(2):117-120. https://doi.org/10.1016/01660934(80)90020-8

21. Asanzhanova NN, Ryskeldinova SZ, Chervyakova OV et al (2017) Comparison of different methods of purification and concentration in production of influenza vaccine. Bull Exp Biol Med 164(2):229-232. https://doi.org/10.1007/s10517-017-3964-y

22. Ferko B, Stasakova J, Romanova J et al (2004) Immunogenicity and protection efficacy of replication-deficient influenza A viruses with altered NS1 genes. J Virol 78(23):13037-13045. https://doi. org/10.1128/JVI.78.23.13037-13045.2004

23. Lin J, Zhang J, Dong X et al (2006) Safety and immunogenicity of an inactivated adjuvanted whole-virion influenza A (H5N1) vaccine: a phase I randomised controlled trial. Lancet 368(9540):991997. https://doi.org/10.1016/S0140-6736(06)69294-5

24. Luke CJ, Subbaro K (2006) Vaccines for pandemic influenza. Emerg Infect Dis 12(1):66-72. https://doi.org/10.3201/eid12 01.051147

25. Palese $P$ (2006) Making better influenza virus vaccines? Emerg Infect Dis 12(1):61-65. https://doi.org/10.3201/eid1201.051043

26. Horimoto T, Takada A, Fujii K et al (2006) The development and characterization of $\mathrm{H} 5$ influenza virus vaccines derived from a 2003 human isolate. Vaccine 24(17):3669-3676. https://doi. org/10.1016/j.vaccine.2005.07.005

27. De Jonge J, Isakova-Sivak I, Van Dijken H et al (2016) H7N9 live attenuated influenza vaccine is highly immunogenic, prevents virus replication, and protects against severe bronchopneumonia in ferrets. Mol Ther 24(5):991-1002. https://doi.org/10.1038/ mt. 2016.23

28. Baz M, Boonnak K, Paskel M et al (2015) Nonreplicating influenza A virus vaccines confer broad protection against lethal challenge. MBio 6(5):01487-15. https://doi.org/10.1128/mBio.01487 $-15$

29. Music N, Reber AJ, Kim MC et al (2016) Supplementation of H1N1pdm09 split vaccine with heterologous tandem repeat M2e5x virus-like particles confers improved cross-protection in ferrets. Vaccine 34(4):466-473. https://doi.org/10.1016/j.vacci ne.2015.12.023

30. Baker SF, Guo H, Albrecht RA et al (2013) Protection against lethal influenza with a viral mimic. J Virol 87(15):8591-8605. https://doi.org/10.1128/JVI.01081-13

31. Maher JA, DeStefano J (2004) The ferret: an animal model to study influenza virus. Lab Anim (NY) 33(9):50-53. https://doi. org/10.1038/laban1004-50
32. Bosch BJ, Bodewes R, de Vries RP et al (2010) Recombinant soluble, multimeric HA and NA exhibit distinctive types of protection against pandemic swine-origin 2009 A (H1N1) influenza virus infection in ferrets. J Virol 84(19):10366-10374. https://doi. org/10.1128/JVI.01035-10

33. Lipatov AS, Webby RJ, Govorkova EA et al (2005) Efficacy of $\mathrm{H} 5$ influenza vaccines produced by reverse genetics in a lethal mouse model. J Infect Dis 191(8):1216-1220. https://doi. org/10.1086/428951

34. Johansen K, Nicoll A, Ciancio BC et al (2009) Pandemic influenza A (H1N1) 2009 vaccines in the European Union. Euro Surveill 14(41):19361

35. Kassenov MM, Khairullin BM, Kydyrbayev ZhK et al (2010) Influenza monitoring among birds in Kostanaiskaya Oblast, Republic of Kazakhstan. Agric Biol J N Am 1(5):737-739. https ://doi.org/10.5251/abjna.2010.1.5.737.739

36. Katz J, Hancock K, Veguilla V et al (2009) Serum cross-reactive antibody response to a novel influenza A (H1N1) virus after vaccination with seasonal influenza vaccine. MMWR Morb Mortal Wkly Rep 58(19):521-524

37. Liu G, Song L, Reiserova L et al (2012) Flagellin-HA vaccines protect ferrets and mice against $\mathrm{H} 5 \mathrm{~N} 1$ highly pathogenic avian influenza virus (HPAIV) infections. Vaccine 30(48):6833-6838. https://doi.org/10.1016/j.vaccine.2012.09.013

38. Ninomiya A, Imai M, Tashiro $M$ et al (2007) Inactivated influenza H5N1 whole-virus vaccine with aluminum adjuvant induces homologous and heterologous protective immunities against lethal challenge with highly pathogenic $\mathrm{H} 5 \mathrm{~N} 1$ avian influenza viruses in a mouse model. Vaccine 25(18):3554-3560. https:// doi.org/10.1016/j.vaccine.2007.01.083

39. Schultze V, D'Agosto V, Wack A et al (2008) Safety of MF59 ${ }^{\mathrm{TM}}$ adjuvant. Vaccine 26(26):3209-3222. https://doi.org/10.1016/j. vaccine.2008.03.093

40. Tabynov K, Kydyrbayev Zh, Sansyzbay A et al (2012) Immunogenic and protective properties of the first kazakhstan vaccine against pandemic influenza A (H1N1) pdm09 in ferrets. Virol Sin 27(6):344-351. https://doi.org/10.1007/s12250-012-3272-7

Publisher's Note Springer Nature remains neutral with regard to jurisdictional claims in published maps and institutional affiliations. 